\title{
Societal Dimensions
}

National Cancer Institute

\section{Source}

National Cancer Institute. Societal Dimensions. NCI Thesaurus. Code C53676.

Various research and other activities that address the broad implications of nanotechnology to society, including benefits and risks, such as: 1) Research directed at environmental, health, and safety impacts of nanotechnology development and risk assessment of such impacts; and 2) Research directed at identifying and quantifying the broad implications of nanotechnology for society, including social, economic, workforce, educational, ethical, and legal implications. 